\title{
LA DISCIPLINA OPERATIVA Y LA MICROEMPRESA ${ }^{1}$ \\ Francisca Rosales Gómez ${ }^{2}$ \\ José Luis Sánchez Leyva ${ }^{3}$ \\ José Antonio Vergara Camaho ${ }^{4}$ \\ Oreste Pimentel Reyes ${ }^{5}$
}

Para citar este artículo: Rosales, F., Sánchez J., Vergara J. y Pimentel O. (2015). "La disciplina operativa y la microempresa". Inquietud Empresarial. Vol. XV (1), pp. 77-106

Fecha de recepción: 30 de noviembre de 2014

Fecha de aceptación: 25 de marzo de 2015

\footnotetext{
1 Presentación de los resultados de un trabajo de investigación sobre la disciplina Operativa en Coatzacoalcos, realizado en la ciudad de Coatzacoalcos, Veracruz, México.

2 Doctora en Gobierno y Administración Pública, profesora de tiempo completo de la Facultad de Contaduría y Administración de la Universidad Veracruzana, Campus Coatzacoalcos, Veracruz, México. frosales@uv.mx

3 Doctorado en Gobierno y Administración Pública. , Maestría en Gobierno y Asuntos Públicos. Licenciatura en Administración de Empresas en la Universidad Veracruzana. luissanchez01@uv.mx

4 Doctorado en Educación en la Escuela Libre de Ciencias Políticas y Administración Pública. Maestría en Tecnología Educativa en la Universidad Autónoma de Tamaulipas. Licenciatura en informática en la Universidad Veracruzana.jvergara@uv.mx

5 Estudiante de la Facultad de Contaduría y Administración de la Universidad Veracruzana, Campus Coatzacoalcos.killers@hotmail.com
} 


\section{RESUMEN}

El objetivo de este estudio es identificar los elementos de la Disciplina Operativa en las actividades empresariales de las microempresas en Coatzacoalcos, México. Para su análisis se diseña y aplica un instrumento a 20 empresas que cumplen las recomendaciones sugeridas en la investigación como la precaución en el uso de materiales peligrosos, uso de equipos con especificaciones sencillas y de fácil acceso a sus empleados, seguimiento de alguna norma para identificar riesgos, buen manejo medio ambiental, entre otros. Se concluye que a pesar de los hallazgos encontrados en la aplicación de la metodología de la Disciplina Operativa, en general se adolece de muchas prácticas importantes, además se puede considerar que las empresas de construcción de la ciudad de Coatzacoalcos no tienen los cuidados ambientales obligatorios para esta actividad.

\section{PALABRAS CLAVE}

Disciplina Operativa, Disponibilidad, Cumplimiento, Comunicación, Calidad

\section{Operational discipline and microenterprises}

\section{ABSTRACT}

The aim of this study is to identify the elements of the Operational Discipline in the business of micro-enterprises in Coatzacoalcos, Mexico. For the analysis an instrument is designed and applied to 20 companies which fulfill the suggested recommendations in research such as caution in the use of hazardous materials, use of equipment with simple specifications and easily accessible to employees, monitoring of some standard to identify risks, good environmental management, among others. We conclude that despite the findings in the application of the methodology of Operational Discipline, it is generally suffers from many important practices, and can be considered as construction companies of the city of Coatzacoalcos no mandatory environmental care this activity.

\section{KEY WORDS:}

Operational Discipline, Availability, Performance, Communication, Quality 


\section{A disciplina operativa e a microempresa}

\section{RESUMO}

O objetivo deste estudo é identificar os elementos da Disciplina Operativa nas atividades empresariais das microempresas em Coatzacoalcos, México. Para sua análises desenha e aplica um instrumento a 20 empresas que cumprem as recomendações sugeridas na investigação como a precaução no uso de materiais perigosos, uso de equipamentos com especificações simples e de fácil acesso a seus empregados, seguimento de alguma norma para identificar riscos, bom manejo do meio ambiente, entre outros. Se conclui que apesar dos achados encontrados na aplicação da metodologia da Disciplina Operativa, em geral sofre em muitas práticas importantes, além de que se pode considerar que as empresas de construção da cidade de Coatzacoalcos não tem os cuidados ambiientais obrigatórios para esta atividade.

\section{PALAVRAS CHAVE}

Disciplina Operativa, Disponibilidade, Cumprimento, Comunicação, Qualidade

\section{INTRODUCCIÓN}

Hoy en día la globalización de los negocios ha causado una alta competitividad en los mercados, exigiendo a las empresas productos y servicios con mayor calidad, por lo cual muchas de éstas tienen que estructurar metodológicamente sus actividades y procesos, para obtener un producto de calidad que les permita obtener ganancias y abrirse campo dentro del mercado, si queremos apoyar a las microempresas en su desarrollo y permanencia en el mercado al mundo de los negocios es importante conocer en qué consiste la disciplina operativa, la cual nos servirá para proponer medidas para la ordenación de sus actividades.

La disciplina es un entrenamiento que corrige, moldea, genera fortaleza y perfecciona su misión para formarte de buenos hábitos (León, 2010). Esta expresión es utilizada para referirse a una acción para lograr comportarse de manera determinada.

Como las empresas micro, pequeñas y medianas (MIPYMES), se originan 
por una parte porque existen emprendedores que deciden invertir sus pequeños ahorros, por la otra, hay un número significativo de trabajadores que al verse sin la fuente de trabajo por haber sido liquidados se convierten en comerciantes, por su puesto en su gran mayoría carecen del don de la administración y del mando.

Hoy más que nunca en México todas las empresas, cualquiera que sea su tamaño y sector de actividad, se ven forzadas a competir en un entorno global por lo que las micro, pequeñas y medianas empresas (MIPYMES) representan un sector lleno de vida y de importancia en el país.

El periódico "El Universal" informó que de acuerdo con el Director del INEGI, Eduardo Sojo, el 95.2\% (4.8 millones) de las empresas en México son Microempresas y dan empleo al $45.6 \%$ de todos los trabajadores. (El Universal, 2010)

Por lo que se reconoce que las MIPYMES son importantes para la economía y para el desarrollo del país, debido al número de empleos que generan y por la contribución en la distribución social de la riqueza.

En México según datos de INEGI 2000, sólo un 20\% de las MIPYMES cuenta con algún tipo de certificación, como ISO-9000, industria limpia u otro tipo. Asimismo, aún la certificación más común -el ISO 9000-es utilizado sólo por el $10 \%$ de las empresas del sector. Los porcentajes para las otras versiones de ISO apenas llegan al $2 \%$ (en el caso del ISO 9002). La información refleja un bajo interés de las MIPYMES mexicanas por adoptar este tipo de esquemas, como se puede apreciar en los siguientes datos: las MIPYMES utilizan técnicas de mejora de calidad o productividad, como las relacionadas con administración de calidad total 32\%, control estadístico de procesos y equipos de control $25 \%$.

Como podemos ver hay un bajo porcentaje de empresas que poseen certificaciones; la metodología de justo a tiempo es poco usada en las empresas (apenas una de cada diez empresas la ha implementado). Finalmente, el 24\% de las MIPYMES maneja algún tipo de licencia o patente, del cual el $39 \%$ pertenece al sector manufactura, el $17 \%$ a servicios y el $16 \%$ al comercio, cabe aclarar que dentro de este esquema se incluyen algunos micro-negocios que por necesidad de la exigencia del mercado se ven obligados a certificarse. 


\section{Empresas Familiares}

Las Empresas Familiares pueden ser micro, pequeñas, medianas y grandes empresas.

Definimos los Negocios Familiares como las unidades de producción de bienes o prestación de servicios, operadas por los miembros de una familia y personas contratadas por la familia, que dependen de los ingresos que se conciben en el Negocio Familiar. La entrada a este sistema es por nacimiento, y la membrecía es permanente.

Tratándose de parientes, la relación laboral entre ellos es más bien informal, de igual manera se entiende que, un negocio familiar es cualquier negocio en el cual la mayor parte de la propiedad o del control forma parte del patrimonio familiar, y están involucrados directamente dos o más miembros de la familia.

Es un sistema dual complejo cuyos elementos son la familia y el negocio; es decir, los miembros de la familia implicados en el negocio son parte de un sistema de negocios y, a la vez, parte de un sistema familiar. Cuando estos dos sistemas se superponen es donde el conflicto puede ocurrir, porque cada sistema tiene sus propias reglas, funciones y requisitos. Por ejemplo, el sistema de la familia es emocional, donde afloran algunos aspectos subjetivos, en tanto que en la empresa las relaciones son de tipo técnico laboral, donde prevalece la eficiencia y lealtad.

La función que un miembro tiene en la familia -esposo/padre, esposa/madre, hijo/hermano/hermana, conlleva ciertas responsabilidades y expectativas. Además, las familias tienen su propio estilo de comunicación y de resolver conflictos, que han ido desarrollando a través de los años. Estos estilos pueden ser buenos para situaciones familiares, pero pueden no ser los mejores para resolver problemas dentro de la empresa.

Las empresas familiares han sido objeto de estudio e investigación desde distintas formas y perspectivas. Tópicos como liderazgo, problemas de sucesión, estrategia, transferencia de poder, innovación entre otros han sido temas elegidos por los investigadores, y abordados desde hace algún tiempo. 
Nos interesa en este estudio proponer la técnica de la Disciplina Operativa en el desarrollo de las Microempresas de Coatzacoalcos, sean o no de tipo familiar.

\section{La Disciplina Operativa}

Es un método administrativo que se propone mejorar los procesos de una empresa. Se logra a través del control y administración de tres grandes elementos: la tecnología, las instalaciones y el personal (Obregón, 2007)

Disciplina Operativa, nació en México en 1983, para fortalecer el desempeño de Seguridad, Salud y Protección Ambiental (SSPA). El concepto de Disciplina Operativa, fue posteriormente utilizado en todos los Giros del Negocio, para fomentar y fortalecer la Excelencia Operacional.

La disciplina operativa es aquella encargada de vigilar que todos los procedimientos sean realizados adecuadamente, logrando que estos sean de calidad. Ayuda a que los procedimientos (operacionales, administrativos, de mantenimiento), sean más confiable, así mismo lograr disminuir los incidentes que represente un riesgo. (ArcaCorporat)

De acuerdo con Petróleos Mexicanos, es el "cumplimiento riguroso y continuo de todos los procedimientos e instrucciones de trabajo, a través del proceso de tenerlos disponibles con la mejor calidad y cumplimiento, comunicarlos de forma efectiva a quienes los aplican y de exigir su apego estricto". (Pemex, 2010).

De acuerdo con Alvarado (2012) la definición e implementación de las mejores normas operativas mediante el cumplimiento riguroso de un proceso uniforme de trabajo, asegura la máxima eficiencia en los equipos, debido a que está tiene como objetivo el gestionar la calidad aplicada en los procedimientos, logrando con esto, la creación de estándares que permitan evaluar los resultados de manera más eficiente, segura y con ello conseguir una retroalimentación que contribuya a una mejora continua. (arcacorporat)

Es parte integral de los procedimientos, de las actividades diarias y también de la manera de cómo la compañía enfoca sus iniciativas. No hay atajos y nada reemplaza los esfuerzos intensivos necesarios para sostener los 
procesos. (Dyer, 2005) Según Miranda (2010) Entre las diversas causas que propician el incumplimiento en la aplicación de procedimientos destacan:

- Limitada disponibilidad en las áreas donde se requiere hacer uso de ellos

- Falta de uniformidad en la forma y contenido de la documentación

- Procedimientos incompletos y/o extensos

- Procedimientos con un alto grado de complejidad

- Falta de claridad

- Obsolescencia (no refleja las condiciones o instalaciones actuales

- Desconocimiento, falta de comprensión por parte del personal

- Falta de difusión, capacitación y entrenamiento al personal

- Falta de consistencia en su aplicación

Es una metodología sumamente útil y accesible que brinda la retroalimentación continua y nos permite la participación de todo el personal, para optimizar la forma de realizar actividades. (PEMEX, 2011).

Es decir dentro de la empresa todos hacen lo correcto, todas las veces. Las empresas de clase mundial son todas aquellas empresas que pueden competir en términos de eficiencia y calidad con cualquier otra en el mundo. Se observa que estas definiciones van de la mano, ya que las empresas capacitan su personal de manera que hagan los procesos y/o actividades correctamente, estas acciones se identifican de alguna manera con los principios de clase mundial de ahí que posiblemente se puedan convertir en empresas altamente competitivas y por consiguiente en "empresas de clase mundial"

Estos dos términos conjugados, desarrollan una nueva forma de llevar la disciplina operativa a las empresas convirtiéndolas de clase mundial. Cuando una organización requiere de modificaciones, lo debe de hacer con la intención de mejorar sus procesos y procedimientos, es aquí donde surge la idea del cómo hacer posible tales cambios sin perjudicar a la totalidad de la empresa. 
Muchos son los beneficios que trae consigo la D.O, una de ellas es que el recurso humano se vuelve más capaz y competitivo en su ámbito laboral, y con esto mejora la eficiencia de la empresa, dando así a sus clientes un servicio o productos de calidad.

Pemex presenta una metodología sumamente útil y accesible que brinda retroalimentación continua, eso les permite la participación de todo el personal, para uniformizar la manera de realizar actividades.

\section{Recursos Humanos}

La Disciplina Operativa según (Universidad Catolica de Colombia, 2003) toda empresa debe tomar en cuenta para ejecutar sus diversos planeamientos, ya que de acuerdo a la organización y procedimientos que utilicen se generan los resultados que pretenden alcanzar. Como resultado de este seguimiento es indispensable involucrar al recurso humano el cual es indispensable entre las directrices y el empoderamiento de nuestras metas, además es necesario contar con líderes que comprometan el esfuerzo común de la empresa para lograr engrandecerla y construir los procesos que enfoquen proyectos para mejorar la calidad.

Se hace un análisis de funcionamiento de la empresa a la luz de los conocimientos de la teoría, de los sistemas de producción para mostrar que es posible aplicar a la construcción. Esto se logra al presentar el enfoque de sistemas, luego se establece la necesidad de que la empresa realice las tres funciones principales de la administración y la conveniencia de realizar algunas subfunciones. Después de visualizan las actividades de producción de la empresa, bajo la teoría de las operaciones y las implicaciones que tiene esta actividad, finalmente se hace una caracterización de la gerencia de operaciones en una empresa, tomando en consideración el nivel y tipo de decisiones que debe tomar, y los criterios que puede emplear para valorar el desempeño de las operaciones (Arcudia Abad, Pech Pérez y Álvarez Romero, 2005).

La disciplina operativa es aplicada por los altos ejecutivos, y profesionales de las operaciones y la seguridad, incluidos los directores de las operaciones, ejecutivos de seguridad, gerentes de plantas o instalaciones y unidad de operaciones, jefes de seguridad de obras. (Dupont, 2014), Directores, gerentes, jefes de área, supervisores, cabos de cuadrilla, personal operativo 
y manual, tanto de operación, mantenimiento, construcción, planeación y finanzas, ventas, adquisiciones almacén, personal administrativo, todos sin excepción, deben conocer el proceso, y aplicar correctamente todos los procedimientos, solo así se alcanza la excelencia. (Arca Corporate), todo el recurso humano, ya que es el único capaz de implantar los sistemas para la mejora de los procesos y de aplicar la tecnología que nos demanda la sociedad (PEMEX, 2011).

El objetivo del proceso de disciplina operativa es asegurar que las actividades/operaciones, sean llevadas a cabo y consistentemente a través de sus cuatro etapas.

Como todo modelo de trabajo, la Disciplina Operativa debe planearse. En su etapa de planeación se deben formar los equipos de trabajo, quienes a su vez les corresponderán elaborar sus planes y programas de trabajo.

\section{Capacitación}

En México, la capacitación del trabajador es obligatoria y deberá de realizarse en horario de trabajo, menciona la Ley Federal del Trabajo, salvo que por necesidades de servicio se modifique. Se le enterará de los días y número de capacitaciones a las que tendrá que acudir, en caso contrario, se entenderá que podrá rescindirse el contrato donde se señala la obligatoriedad de la instrucción del trabajador.

En la actualidad la decisión sobre el diseño óptimo de los puestos de trabajo y la elección de los objetivos han quedado casi totalmente en manos de los directivos y especialistas en tecnología, así (Del Valle, 2010) dice: Las empresas y las organizaciones en general funcionan impulsadas por una serie de elementos que en economía se denominan factores de producción. Éstos son de diversa índole, a saber: materias primas, recursos energéticos, maquinaria, recursos humanos, etc. Su importancia varía según la actividad y la tecnología utilizada; pero en toda organización, cualquiera que sea su naturaleza, existe un elemento imprescindible sin el cual la marcha de la misma es impensable: este factor crucial e insustituible es el personal.

\section{Administración del cambio}

Administración del Cambio es el proceso, las herramientas y las técnicas para administrar desde el lado de las personas el cambio en procesos, 
para alcanzar los resultados requeridos, y concretar el cambio de manera efectiva desde el agente individual del cambio, el interior del equipo y la totalidad del sistema. (CACIT Group)

-Proporciona una imagen clara de cómo se verá el negocio después de la transformación.

- Proporciona detalles de lo que hay que hacer durante el proceso de cambio para construir conocimientos y habilidades.

-Desarrolla los planes necesarios para el negocio, las métricas y los resultados.

- Utiliza una variedad de diagnósticos y facilitadores para identificar las actitudes y comportamientos de las personas afectadas por el cambio. (Integrante de Ernst y Young Global, 2012)

\section{Cambio del Comportamiento}

Los programas de cambio centrados en el comportamiento tienden a apoyarse en la inclusión y participación activa de muchos empleados. Un cambio de conducta exitoso mejora los procesos de toma de decisión individual y de grupo, la identificación de problemas, la solución de los mismos, la comunicación, las relaciones de trabajo y otros similares.

Existen cuatro enfoques del cambio organizacional, que se presentan a continuación:

1. Retroalimentación de encuestas: Consiste en recopilar información de integrantes de una organización o grupo de trabajo y en organizar información de forma comprensible y útil.

Debido a su valor en el diagnóstico organizacional, la retroalimentación de encuesta suele utilizarse como parte de programas de cambio en gran escala, a largo plazo, en combinación con otros enfoques y técnicas.

2. Formación de equipos: Es un proceso mediante el cual los integrantes de un grupo de trabajo o equipo diagnostican la forma en que trabajan juntos y planean cambios para mejorar su efectividad.

3. Asesoría de proceso: Es la asesoría brindada por un consejero (capacitado) para ayudar a los integrantes de una organización a percibir, 
comprender y actuar en acontecimientos de los procesos que suceden en el ambiente laboral. Los acontecimientos de proceso son las formas en que los empleados realizan su trabajo, incluido la conducta de las personas en las reuniones; encuentros formales e informales entre empleados en el trabajo y; en general cualquiera de los comportamientos incluidos al desempeñar una tarea.

4. Programas de calidad de vida en el trabajo: Son actividades que una organización lleva a cabo para mejorar las condiciones que afectan la experiencia de un empleado con una organización. Muchos programas de calidad de vida se ocupan de la higiene y seguridad, participación en decisiones, oportunidades para utilizar y desarrollar talentos y habilidades, control sobre el tiempo o lugar de trabajo, entre otros temas.

Los programas se volvieron populares como respuesta a las demandas de los empleados mediante el uso de programas de trabajo alternativos que incluyen (dar a los empleados cierto control sobre sus propios horarios de trabajo), el empleo de medio tiempo, empleos compartidos o el trabajo, en el hogar. (Amoros, s.f)

\section{La tecnología en los procesos}

De acuerdo con Cuesta (1997), las tecnologías de las tareas contemplan los equipos y materiales así como el procedimiento de organización forman los sistemas de trabajo. Estos sistemas de trabajo están actualmente fuertemente basados en la efectividad de los procesos, considerados como un conjunto de actividades que recibe uno o más entradas y crea un producto de valor para el cliente. Pero estos procesos deben estar diseñados y rediseñados, documentados y difundidos de acuerdo con las nuevas tecnologías.

Los procesos siguen su documentación y normatividad que se utilizarán en sus cuatro fases de: Disponibilidad, Calidad, Comunicación y Cumplimiento.

\section{Disponibilidad}

Al consultar el diccionario, la palabra disponer indica que es "colocar, poner las cosas en orden o en la situación necesaria para lograr un fin". A su vez también puede entenderse como "valerse de una persona o cosa 
como si fuera propia".

Los procedimientos son planes por medio de los cuales se establece un método para el manejo de actividades futuras. Consisten en secuencias cronológicas de las acciones requeridas. Son guías de acción, no de pensamiento, en las que se detalla la manera exacta en que deben realizarse ciertas actividades. Es común que los procedimientos crucen las fronteras departamentales. (Koontz y Weihrich, 2003)

Es por ello que las empresas requieren de un manual de procedimientos que es, un instrumento administrativo que apoya el quehacer cotidiano de las diferentes áreas de una empresa. (Mejía, 2005)

El manual de procedimientos cumplirá la función de mostrar los distintos procesos que cumple la empresa y la forma en la que debe ser realizado el proceso. A su vez también muestra las diferentes partes de la empresa que intervienen en un proceso específico.

Pero también una empresa debe enfrentarse a ciertas limitaciones, dado que también será necesario que los procesos que desempeñe la compañía estén disponibles para su uso de las actividades requeridas.

Como nos menciona Petróleos Mexicanos (2012), dentro de la Disciplina Operativa, la etapa de disponibilidad tiene como propósito asegurar que todos los procedimientos que son requeridos para las actividades estén disponibles y accesibles. A lo cual esta etapa cumplirá los siguientes objetivos:

- Tener una adecuada cobertura de procedimientos para cada una de las actividades que los requieran.

- Contar con información correcta y consistente en todas las áreas y/o departamentos.

- Definir claramente prioridades sobre la base del riego e impacto que tengan los procedimientos para establecer criterios de revisión.

Para satisfacer de manera óptima las necesidades, requerimientos y expectativas del cliente, las organizaciones eficientes crean y utilizan sistemas de calidad. Los sistemas de administración de la calidad conjuntan los ingredientes necesarios para los empleados de la organización para que 
puedan identificar, diseñar, producir, entregar y apoyar los productos y servicios que el cliente desea. (Lacasa, 2007)

Las normas ISO 9000 son una serie de normas para el aseguramiento de la calidad, que son aceptadas alrededor del mundo. Su importancia radica en que, cuando se adquiere un producto o servicio de una empresa esta se encuentra registrado en ISO 9000, se tiene la seguridad de que la calidad que se está recibiendo será como se esperaba. (Portal de Educación Básica, 1999)

Los principios de gestión de la calidad, de acuerdo con lo indicado en la norma ISO 9001 son:

1.- Enfoque al cliente: las organizaciones dependen de sus clientes, por lo tanto deben comprender sus necesidades actuales y futuras, satisfacer sus requisitos y esforzarse en exceder sus expectativas.

2.- Liderazgo: los líderes establecen la unidad de propósito y la orientación de la organización. Deben crear y mantener un ambiente interno, en el cual el personal pueda llegar a involucrarse en el logro de los objetivos de la organización.

3.- Participación del personal: El personal, a todos los niveles, es la esencia de la organización, y su total compromiso posibilita que sus habilidades sean usadas para el beneficio de ésta.

4.- Enfoque basado en procesos: Un resultado deseado se alcanza más eficientemente cuando las actividades y los recursos relacionados se gestionan como un proceso.

5.- Enfoque de sistema para la gestión: identificar, entender y gestionar los procesos interrelacionados como un sistema, contribuye a la eficacia y eficiencia de la organización en el logro de sus objetivos.

6.- Mejora continua: la mejora continua del desempeño global de la organización, debe de ser un objetivo permanente.

7.- Enfoque basado en hechos para la toma de decisiones: las decisiones eficaces se basan en el análisis de los datos y en la información previa. 
8.- Relaciones mutuamente beneficiosas con el proveedor: una organización y sus proveedores son interdependientes, y una relación mutuamente beneficiosa aumenta la capacidad de ambos para crear valor.

Los emprendedores invierten estableciendo microempresas con el objetivo de establecerse y permanecer en los mercados, piensan expandir sus ventas, adquirir recursos y diversificarse.

\section{Comunicación}

La comunicación es, para empresas e instituciones, una herramienta indiscutible de gestión, ya que permite desarrollar todos los aspectos comerciales y de imagen. Ahora bien, este desarrollo debe realizarse estratégicamente, es decir, con unos objetivos prefijados y unas operativas adecuadas tras un análisis pormenorizado de la situación empresarial y entorno. (Lacasa, 2007)

La comunicación es una actividad consustancial a la vida de la organización, es "la red que se teje entre los elementos de una organización y que brinda su característica esencial: la de ser un sistema" (Katz y Khan, 1986), "el cemento que mantiene unidas las unidades de una organización" (Lucas Marín, 1997), el alma o "el sistema nervioso de la empresa" (Puchol, 1997).

En la comunicación empresarial, la percepción que tengan los receptores es uno de los aspectos más importantes, ya que de ello depende la comprensión y la actitud que tomarán, lo cual repercutirá en la respuesta al mensaje y la forma de retrocomunicación que originará. Son los elementos de referencia, dados por la visión y la misión de la organización, los que tienen que conocer todos los empleados desde los niveles altos de la estructura organizacional hasta los empleados a nivel operacional, lo que facilitará que comprendan cuál es el objetivo, las metas y las tareas que se desarrollan en la organización en todos los niveles. (Sanabria, 2003)

\section{Cumplimiento}

El diccionario define el cumplimiento como la realización de un deber o de una obligación.

La palabra responsabilidad es definida en el diccionario como el cumplimiento de las obligaciones o cuidado al hacer o decidir algo. 
La responsabilidad está entendida en muchas ocasiones como la adquisición de compromisos por parte de la empresa con los diferentes grupos de intereses (los que la empresa considera importantes en cada uno de los casos), y por tanto, como una auto obligación de la empresa a cumplir los objetivos marcados, un compromiso de medir e informar sobre los progresos de la empresa. (Ripollés)

Por lo tanto, cuando se habla de cumplimiento dentro de una empresa se refiere a la obligación o responsabilidad que tiene la misma de finalizar algún compromiso o proceso que a la que se haya accedido. La empresa u organización que logre el cumplimiento de sus compromisos internos y externos logrará que se le considere como una empresa responsable.

Petróleos Mexicanos (2012) nos menciona que la etapa de cumplimiento de la Disciplina Operativa tiene como propósito asegurar el riguroso y continúo acatamiento de los procedimientos. Además, éste debe cumplir con los siguientes objetivos dentro de la organización:

- Contar con medios y sistemas para detectar las desviaciones en el cumplimiento de los procedimientos.

- Analizar las desviaciones para determinar causas y con base en ello mejorar continuamente.

- Desarrollar un alto sentido de compromiso hacia la Disciplina Operativa en todo el personal.

Es un hecho que la sobrevivencia de las empresas, en un entorno de continuos y vertiginosos cambios, depende principalmente de su capacidad de adaptación y su destreza para enfrentar el aumento de la intensidad y diversidad de la competencia. (Vergara, 2009)

La Disciplina Operativa es el cumplimiento riguroso y continuo de todos los procedimientos e instrucciones de trabajo tanto operativos como administrativos y de mantenimiento de un centro de trabajo, a través del proceso de tenerlos disponibles con la mejor calidad y cumplimiento, comunicarlos de forma efectiva a quienes lo aplican y de exigir su apego estricto. (U Virtual, 2014 p. 6)

De acuerdo con la Mtra. Alejandra (2014) dice que la disciplina operativa debe tener un cumplimiento de los compromisos, detectar las desviaciones 
y retroalimentarse, asumir una buena comunicación para tener un mejor mecanismo y matrices de procedimiento, evaluando el entendimiento, con una calidad veraz, con una cobertura técnica y con un lenguaje de calidad y tener una disponibilidad, resguardo, así como un control de las acciones.

En México las empresas que destacan se autonombran Empresas de Clase Mundial o bien Empresas Socialmente Responsables, solo encontramos que Petróleos Mexicanos (PEMEX), aplica la Disciplina Operativa en sus procesos.

En Coatzacoalcos Veracruz existen 293 microempresas inscritas en la Cámara Nacional de Comercio (CANACO) dedicadas a diversos giros, por lo general los dueños de estas microempresas desconocen estrategias administrativas que eleven el nivel de productividad y su crecimiento, por tal motivo sería conveniente que adoptaran la metodología de la disciplina operativa como fundamento para acceder a un posicionamiento efectivo.

\section{Objetivos}

Identificar los elementos de la Disciplina Operativa en las operaciones o procesos de las Microempresas en Coatzacoalcos.

\section{Diseño Metodológico}

El Tipo de estudio de este trabajo es descriptivo ya que identifica la aplicación de la disciplina operativa en un grupo de Microempresas, también es de un tipo de observacional, se seleccionó este estudio, ya que se busca identificar las diferentes características de trabajo de las microempresas, y se basa en muestras pequeñas de la observación de los aspectos de las microempresas. Además se describe las cualidades de estos fenómenos en los negocios estudiados.

La población está conformada por 293 empresas registradas en la Cámara Nacional de Comercio (CANACO) de las cuales se tomó una muestra de 30 microempresas

A través de un cuestionario elaborado con la finalidad de identificar los elementos de la Disciplina Operativa en las operaciones o procesos de las Microempresas en Coatzacoalcos, dicho instrumento está conformado con preguntas sencillas y de respuesta múltiple; se recogió la información en 
un horario de diez horas a catorce horas, procurando siempre la disposición y disponibilidad de tiempo, para contestar por parte de los participantes.

\section{RESULTADOS}

- Tecnología del Proceso

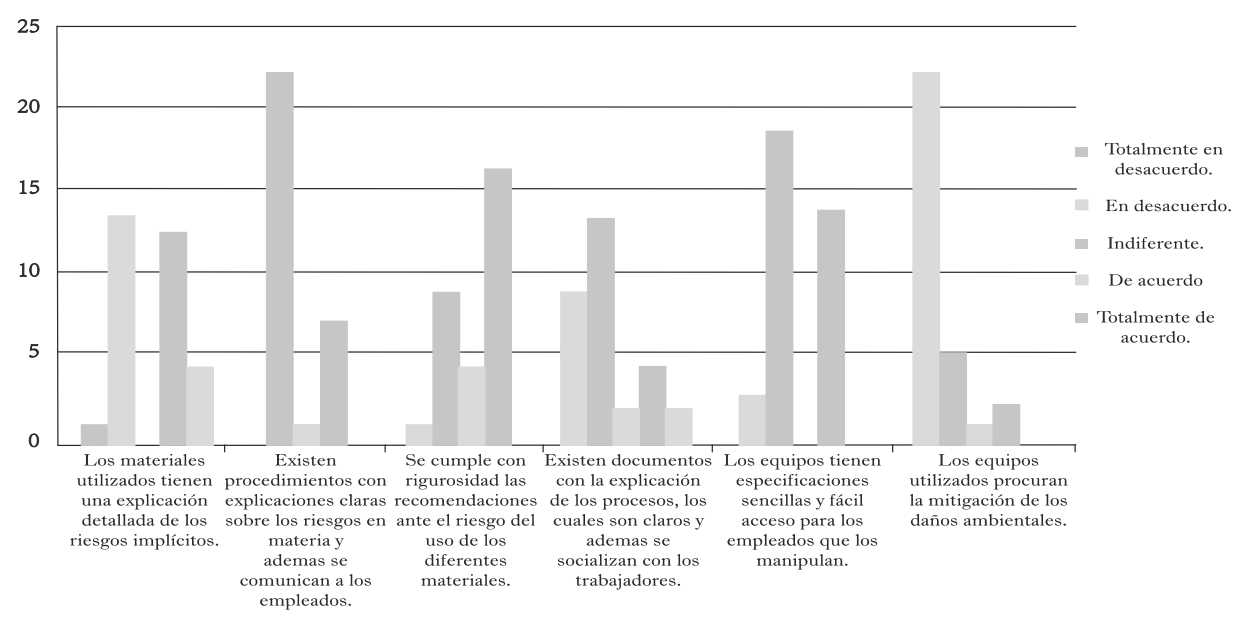

Fuente: Elaboración propia

Con este gráfico se aprecia que 14 empresas de las 30 encuestadas equivalente al $46.6 \%$ no tienen una explicación detallada en sus materiales sobre los posibles riegos que pueda ocurrir, notando también que el $73 \%$ de las empresas no tienen procedimientos con explicaciones sobre los riesgos, pero 20 empresas si cumplen las recomendaciones ante el uso de materiales peligrosos, 22 empresas no tienen documentos con la explicación de los procesos, se aprecia también que el $66.6 \%$ de las empresas tienen equipos con especificaciones sencillas y con acceso a los empleados, desafortunadamente 26 empresas equivalente al $86.6 \%$ utilizan equipos que no procuran la mitigación de los daños ambientales. Se puede notar que las empresas de Coatzacoalcos no poseen una tecnología de proceso adecuado. 
- Análisis del Riesgo del Proceso

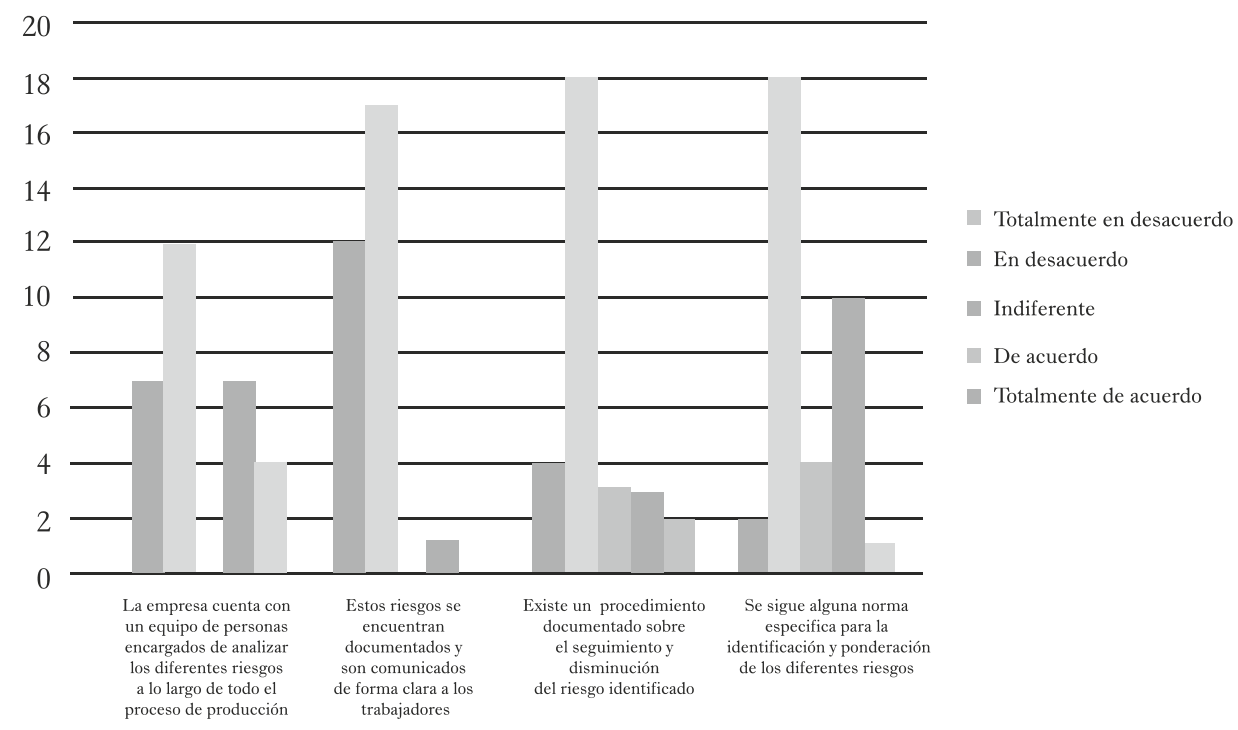

Fuente: Elaboración propia

Con este histograma se puede observar que 12 empresas de las 30 encuestadas equivalente al $63.3 \%$ no tiene personal encargados de analizar los riesgos durante el proceso de producción, el $96.6 \%$ de las empresas no documentan estos riesgos, 22 empresas no tiene procedimientos documentados sobre seguimientos de los riesgos, y el $50 \%$ de las empresas sigue alguna noma para identificar riesgos. Se puede apreciar claramente que las empresas no tienen un análisis de riesgo del proceso. 
- Procedimientos de Operación

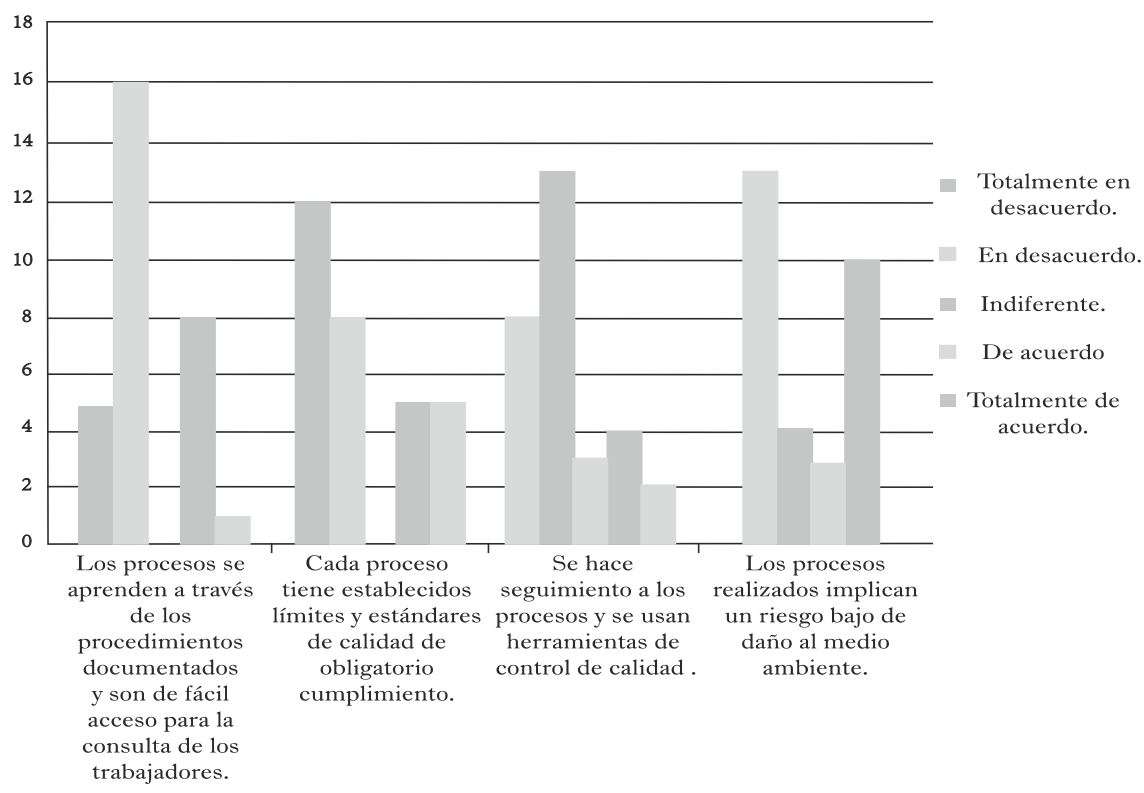

Fuente: Elaboración propia

En este histograma se puede notar que, 21 empresas dicen que los procesos no se aprenden a través de los procedimientos documentados previamente, el $66.6 \%$ de las empresas dijo que los procesos no tiene límites y estándares de calidad establecidos, el $20 \%$ de las empresas hacen seguimiento a los procesos y usan herramientas de control de calidad, desafortunadamente 17 empresas de las 30 dicen que los procesos realizados implican un riesgo bajo de daño al medio ambiente. 
- Normas Generales de Seguridad

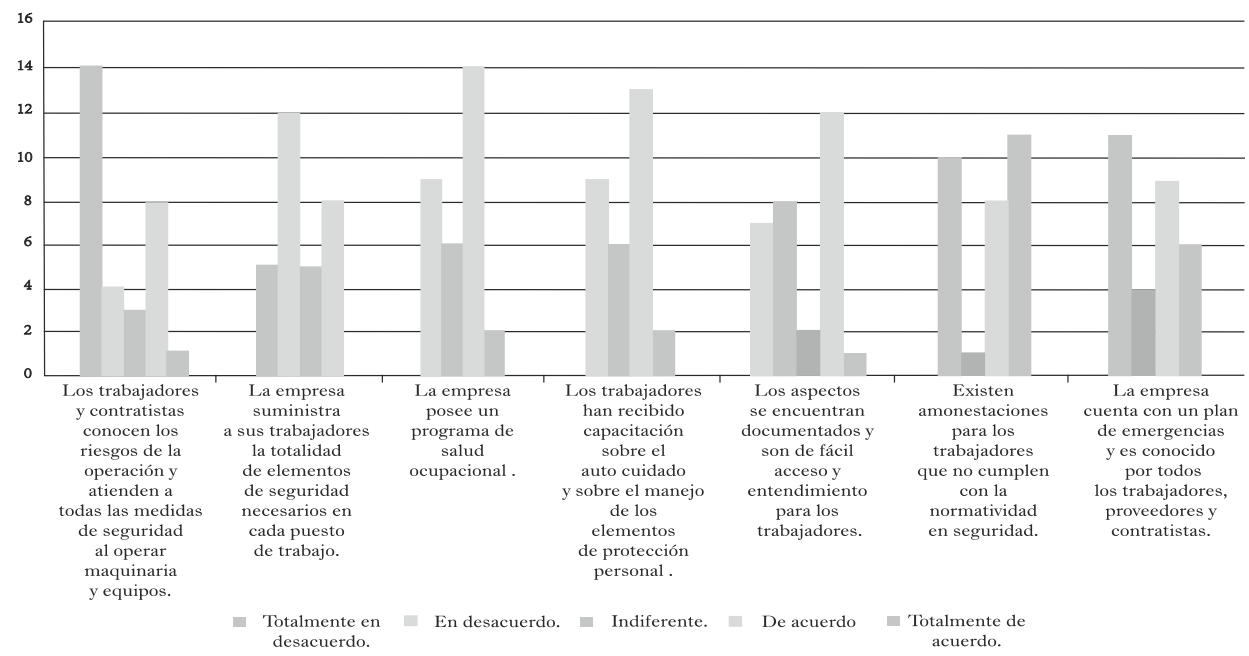

Fuente: Elaboración propia

Con el gráfico se puede apreciar que en el $60 \%$ de las empresas encuestadas sus trabajadores y contratistas no conocen los riesgos de la operación, se ve que solo el $43 \%$ de las empresas suministra a sus trabajadores la totalidad de elementos de seguridad, para los trabajadores que no cumplen con la normatividad en seguridad se refleja un $50 \%$ de los empleados que han recibido capacitación sobre el autocuidado en los elementos de protección personal, se puede apreciar que 19 empresas de 30 dice que existe amonestación, el 50\% de las empresas tienen un plan de emergencia y desafortunadamente el $50 \%$ no tienen un plan establecido. 
- Administración del Cambio

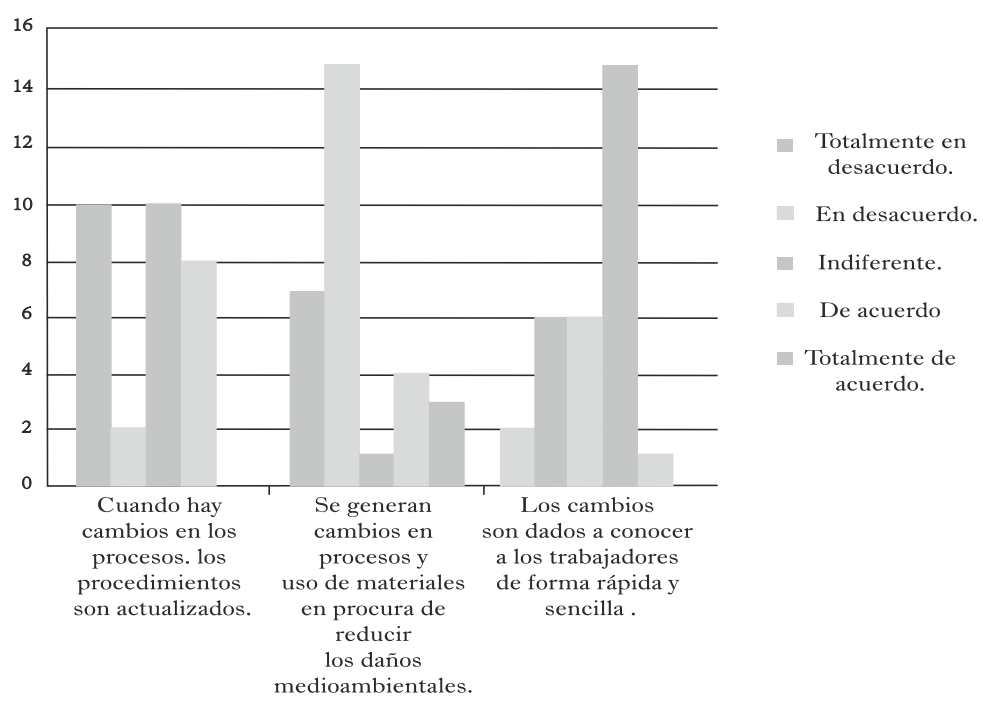

Fuente: Elaboración propia

Con este histograma se puede ver que, 17 empresas de 30 encuestadas equivalentes al $56.6 \%$ dicen que cuando hay cambios en los procesos los procedimientos son actualizados, pero el $33.3 \%$ no actualizan los procedimientos, se puede ver que $73.3 \%$ no procura reducir daños al medio ambiente, mientras que solo 5 empresas verifican esto, el $53.3 \%$ de las empresas dan conocer a los trabajadores los cambios de que surgen pero el $26.6 \%$ no lo concibe. 
- Integridad Mecánica y Aseguramiento de la Calidad

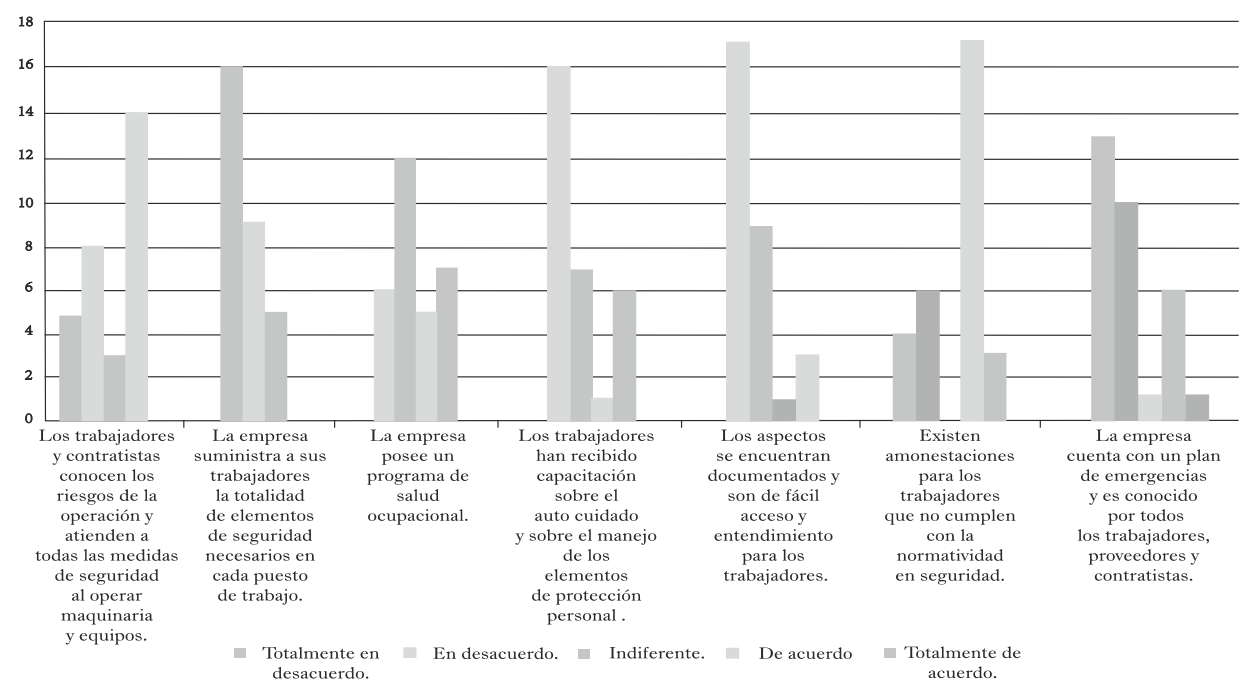

Fuente: Elaboración propia

Con este histograma se puede notar que 14 de 30 equivalente al $46.6 \%$ de las empresas encuestadas dicen que la empresa diseña e implementa programas de mantenimiento para incrementar la seguridad en los trabajadores, el $53.3 \%$ de las empresas en sus instalaciones cuentan con criterios de seguridad y el $16 \%$ no los tienen, 23 empresas equivalentes al $76.6 \%$ argumentan que el mantenimiento de equipos no se hace con criterios de control de calidad, el $66.6 \%$ dice que tienen documentación sobre los procesos de compra y la selección de proveedores, y desafortunadamente el $76.6 \%$ de las empresas no tienen sitios adaptados para el almacenamiento de materiales y la disposición final de los residuos para no afectar el medio ambiente. 
- Capacitación Contínua

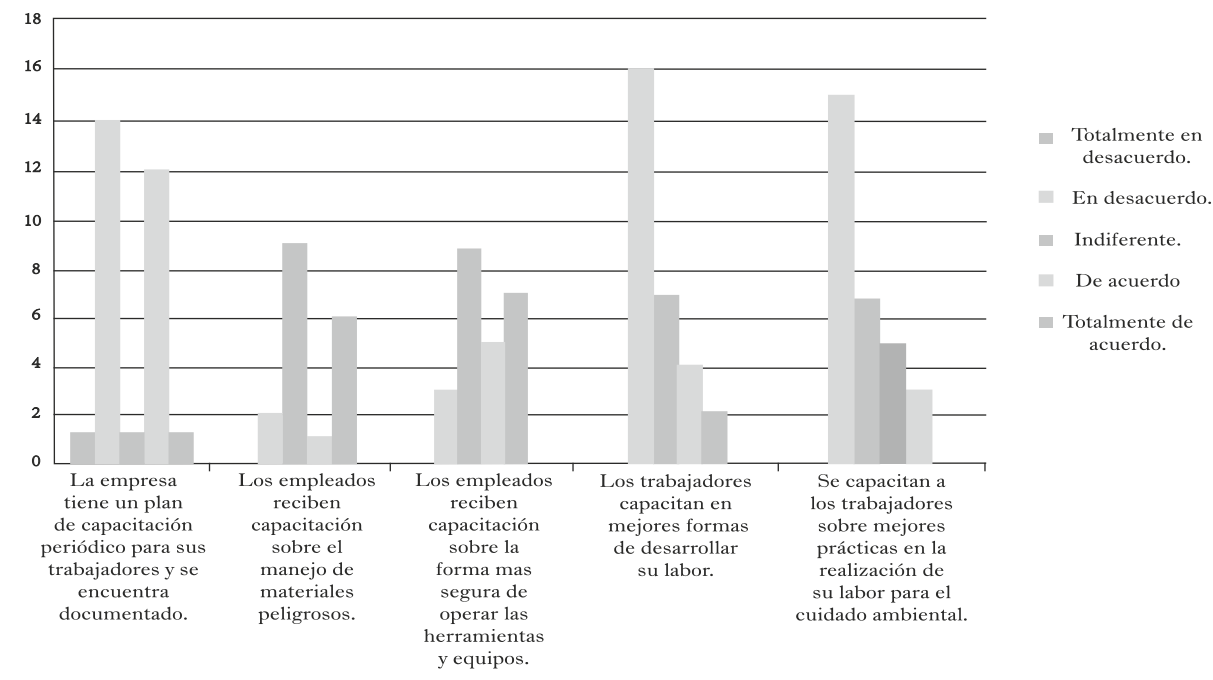

Fuente: Elaboración propia

Con este histograma se puede notar que el $50 \%$ de las empresas encuestadas no tienen un plan de capacitación periódico para sus trabajadores y 14 empresas si los tienen, el $60 \%$ de los empleados reciben capacitación sobre el manejo de los materiales peligrosos, pero 11 de las 30 no reciben capacitación periódica , el $43 \%$ de los empleados reciben capacitación sobre la forma más segura de operar las herramientas, el 76.6 de los empleados no se capacitan en mejores formas de desarrollar su labor, 22 empresas no capacitan a los trabajadores sobre mejores prácticas en la realización de su tarea. Se puede apreciar que no existe una capacitación a los trabajadores en las empresas de construcción de la ciudad de Coatzacoalcos. 
- Análisis y Difusión de Incidentes

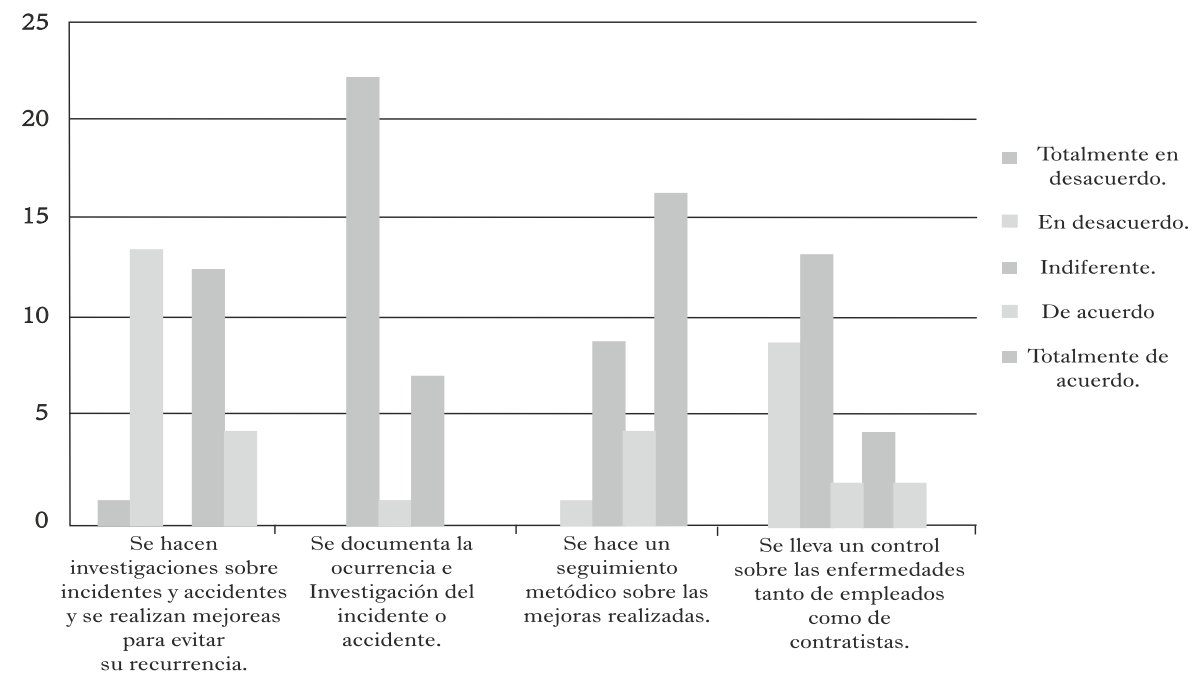

Fuente: Elaboración propia

Con este histograma se puede notar que 24 de las 30 equivalentes al 80 $\%$ del total de las empresas encuestas no realizan investigaciones sobre incidentes y accidentes y solo el $10 \%$ de las empresas si realizan estas investigaciones, el $76.6 \%$ no documenta la ocurrencia del incidente o accidente, 25 empresas no hacen un seguimiento metódico sobre las mejoras y solo el $6 \%$ de las empresas si lo realizan, únicamente el $20 \%$ de las empresas llevan un control sobre las enfermedades tanto de empleados como de contratistas. 
- Contratistas

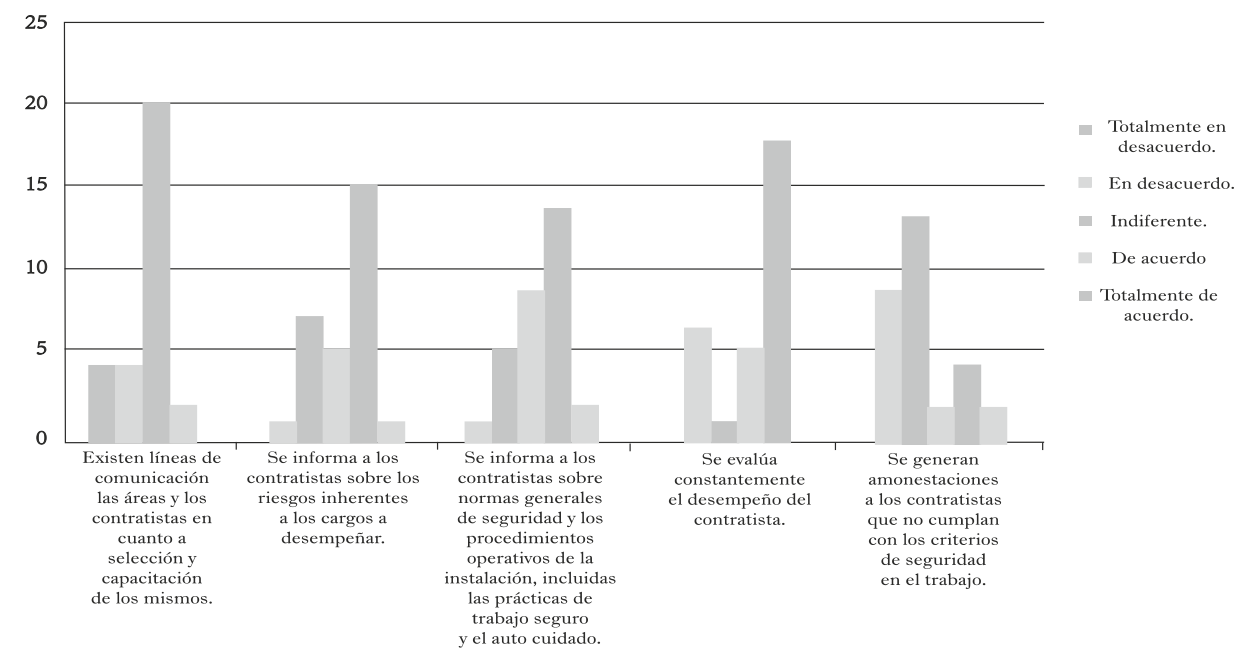

Fuente: Elaboración propia

Con este gráfico se puede apreciar que 22 empresas de las 30 encuestadas equivalentes al $73.3 \%$ tienen líneas de comunicación clara con las áreas y los contratistas, en cuanto a selección y capacitación, $56.6 \%$ de las empresas dicen que se le informa a los contratistas sobre los riesgos a los cargos a desempeñar, el $53.3 \%$ informa a los contratistas sobre las normas generales de seguridad y los procedimientos operativos, 23 de las 30 empresas evalúan constantemente el desempeño del contratista y solo 7 empresas no realizan esta acción, el 70\% generan amonestaciones a los contratistas que no cumplan con los criterios de seguridad. 
- Motivación y Reconocimientos

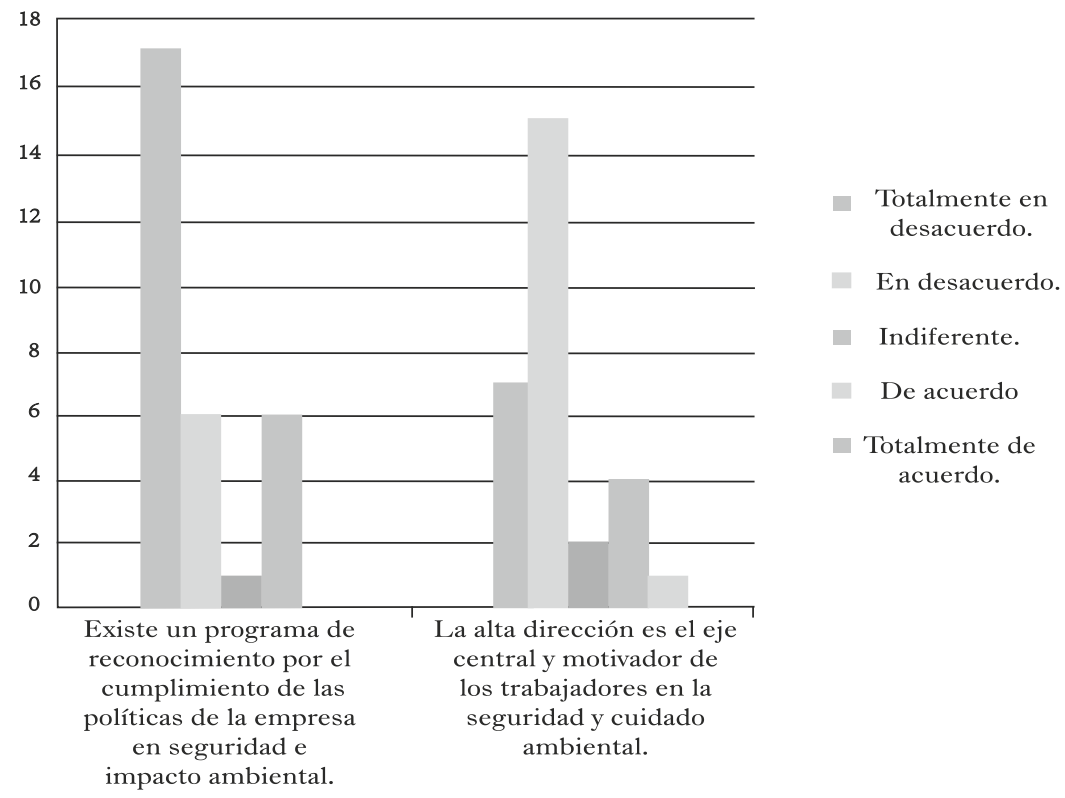

Fuente: Elaboración propia

Con este histograma se puede ver que, 23 empresas de las 30 encuestadas equivalentes al $76.6 \%$ del total les hace falta reconocimientos por el cumplimiento en seguridad e impacto ambiental, el $80 \%$ de la alta dirección de las empresas no tienen motivación hacia los empleados en la seguridad y cuidado ambiental, se puede observar notablemente que en las empresas de construcción de la ciudad de Coatzacoalcos no se tiene ese cuidado ambiental.

\section{DISCUSIÓN Y CONCLUSIONES}

En este estudio se cumple con el objetivo de identificar algunos elementos de la Disciplina Operativa, en la muestra de microempresas, en Coatzacoalcos. Los elementos que se identificaron fueron 20 empresas que cumplen las recomendaciones ante el uso de materiales peligrosos, el $66.6 \%$ de las empresas tienen equipos con especificaciones sencillas 
y con acceso a los empleados, $50 \%$ de las empresas sigue alguna norma para identificar riesgos, 5 empresas procura reducir daños al medio ambiente, $46 \%$ de las empresas encuestadas dicen que la empresa diseña e implementa programas de mantenimiento para incrementar la seguridad en los trabajadores, el 53.3\% de las empresas en sus instalaciones cuentan con criterios de seguridad. El 66.6\% dice que tienen documentación sobre los procesos de compra y selección de proveedores; 14 empresas cuentan con un plan de capacitación periódica para sus trabajadores, el $60 \%$ de los empleados reciben capacitación sobre el manejo de los materiales peligrosos, por lo que se requiere un plan de capacitación periódico para sus trabajadores. Tan solo el 6\% de las empresas lleva un seguimiento metódico sobre las mejoras, únicamente el $20 \%$ de los contratistas; 22 empresas de las 30 encuestadas equivalentes al $73.3 \%$ tienen líneas de comunicación clara con las áreas y los contratistas; $56.6 \%$ de las empresas dicen que se le informa a los contratistas sobre los riesgos a los cargos a desempeñar, el $53.3 \%$ informa a los contratistas sobre las normas generales de seguridad y los procedimientos operativos, 23 de las 30 empresas evalúan constantemente el desempeño del contratista. Estos hallazgos se relacionan directamente con los principios de Disponibilidad, Cumplimiento, Comunicación y Calidad, de la Disciplina Operativa, en virtud de que la disciplina operativa es la encargada de vigilar que todos los procedimientos sean realizados adecuadamente, logrando que estos sean de calidad. En cuanto a las implicaciones prácticas es necesario que los microempresarios de Coatzacoalcos, hagan conciencia en el aprendizaje de nuevas formas de administrar sus negocios, dirigiendo sus esfuerzos a alcanzar las microempresas a pequeñas, las pequeñas a medianas y las medianas a grandes, por otro lado los programas gubernamentales en apoyo a este tipo de empresa, deben estar orientados en este sentido. Este estudio les permite la reflexión en el desempeño de sus actividades empresariales.

El estudio también detectó aquellos elementos de la Disciplina Operativa que no se ponen en práctica en este tipo de microempresas, por ejemplo se revela que las empresas de Coatzacoalcos no poseen una tecnología de proceso adecuado, que no tienen un análisis de riesgo del proceso, 17 empresas de las 30 dicen que los proceso realizados implican un riesgo bajo de daño al medio ambiente, el $76.6 \%$ de las empresas no tienen sitios adaptados para el almacenamiento de materiales y la disposición final de los residuos para no afectar el medio ambiente, el 50\% no tienen un plan establecido, el $53.3 \%$ de las empresas dan conocer a los trabajadores los 
cambios de que surgen pero el $26.6 \%$ no lo hace, y el $76.6 \%$ de las empresas no tienen sitios adaptados para el almacenamiento de materiales y la disposición final de los residuos para no afectar el medio ambiente, el $70 \%$ generan amonestaciones a los contratistas que no cumplan con los criterios de seguridad. Se puede apreciar notablemente que en las empresas de construcción de la ciudad de Coatzacoalcos no se tiene esa atención en el cuidado ambiental.

\section{REFERENCIAS BIBLIOGRÁFICAS}

Amoros, E. (2007). Comportamiento Organizacional. En la búsqueda del desarrollo de ventajas competitivas. Perú: USAT. pp 1-272

Arca Corporate. (s.f.). Arca Corporate. Recuperado 10/03/2014, de Arca Corporate: www.arcacorporate.com.mx/cursos/taler-basico-dedisciplina-operativa/

Arcacorporat. (s.f.). Arcacorporat. Recuperado 18/11/2013, de arcacorporat: http://www.arcacorporate.com.mx/cursos/taller-basico-de-disciplina-operativa/

Arcudia Abad, C., Pech Pérez, J., y Álvarez Romero, S. (2005). La empresa constructora y sus operaciones bajo un enfoque de sistemas. Revista Académica de la Facultad de Ingeniería Universidad Autónoma de Yucatán. Mexico: UADY. pp. 25-36

CACIT Group. (s.f.). Cacit grupo . Obtenido de http://www.cacitgroup. com/vertical/adcambio/adcambio.htm

Dupont. (2014). Recuperado 10/03/2014, de http://www.dupont.mx/productos-y-servicios/consulting_services-process-technologies/ seguridad-laboral-consultoria/usos-y-aplicaciones/taller-disciplina-operacional.html

Dyer, D. (2005). Procter y Gamble (Rising Tide): 165 años construyendo una marca. Boston: Norma.

Ernst y Young Global. (2012). Administración del cambio. Hacemos que las cosas ocurran. Mexico: EY. pp. 1-4 
Koontz, H. y Weihrich, H. (2003). Administracion: Una Perspectiva Global (12 Ed.). México: Mcgraw-Hill.

Lacasa, A. (2007). Gestión de la comunicación empresarial.

León, G. (2010). Valores y Disciplina. México: NL

Mejía, C. (2005). La Guía del Ingeniero Industrial y del Emprendedor. Recuperado 16/09/2014, Universidad Nacional Autónoma de México: http://www.ingenieria.unam.mx/ guiaindustrial/diseno/ info/6/1.htm

Miranda, M. A. (2010). Guia Tecnica. Disciplina Opertaviva. México. pp. $1-16$

Obregón, R. (2007). Implementación de la disciplina operativa en la administración de operaciones en la industria petrolera de Guatemala. Guatemala: USAC. pp. 1-149

Portal de Educación Básica. (1999). La norma ISO 9000 y la competencia laboral. Mexico: SEB. pp. 1-21

Ripollés, F. (s.f.). Jornades de Foment de la Investigació. La empresa responsable. España: Universitat Jaume I. pp. 1-9

S-A. (2007). Gestión de la Comunicación Empresarial.

S-A. (2010). INEGI. El Universal. México.

Sanabria, B. (2003). Módulo Institucional. Comunicación Empresarial (Ejecutivo). Puerto Rico: Universidad de Puerto Rico en Humacao. pp. 1-14

Universidad Virtual. (2010). Proceso de Disciplina Operativa. México: Pemex. pp. 1-12

Universidad Virtual (2011). Auditorias Efectivas. México: Pemex. pp. 1-64

Universidad Virtual. (2012). Proceso de Disciplina Operativa "Disponivilidad". México: Pemex. pp. 1-24

Universidad Virtual. (2012). Proceso de Disciplina Operativa "Cumpliminento". México: Pemex. pp. 1-11 
Universidad Católica de Colombia. (2003). Administración, Pensamiento, Proceso, Estratégia y Vanguardia. Bogotá: Universidad Católica de Colombia.

Vergara, G. (2009). Importancia de la Competitividad Empresarial y Profesional para lograr el éxito. 